\title{
An estimation of the Higgs boson mass in the two loop approximation in a noncommutative differential geometry
}

\author{
Yoshitaka OKumurA * \\ Department of Natural Science, Chubu University, Kasugai, 487, Japan
}

March 31, 2018

\begin{abstract}
In the two loop approximation, a renormalization group analysis of the Higgs boson mass is performed based on the condition $g^{2}=(5 / 3) g^{\prime 2}=4 \lambda$ for $\mathrm{SU}(2)_{\mathrm{L}}, \mathrm{U}(1)_{\mathrm{Y}}$ gauge and the Higgs quartic coupling constants, respectively. This condition is introduced in the new scheme of our noncommutative differential geometry (NCG) for the reconstruction of the standard model. However, contrary to SU(5) GUT without supersymmetry, the grand unification of coupling constants is not realized in this scheme. The physical mass of the Higgs boson depends strongly on the top quark mass $m_{\text {top }}$ through the Yukawa coupling of the top quark in the $\beta$ functions. The two loop effect lowers the numerical value calculated within the one loop approximation by several GeV. The Higgs boson mass varies from $150.25 \mathrm{GeV}$ to $167.15 \mathrm{GeV}$ corresponding to $169.34 \mathrm{GeV} \leq m_{\text {top }} \leq 181.02 \mathrm{GeV}$. We find $m_{\mathrm{H}}=158.18 \mathrm{GeV}$ for $m_{\mathrm{top}}=175.04 \mathrm{GeV}$ and $m_{\mathrm{H}}=166.12 \mathrm{GeV}$ for $m_{\text {top }}=180.34 \mathrm{GeV}$.
\end{abstract}

\section{Introduction}

The Higgs mechanism is essential for any spontaneously broken gauge theory. Its presence ensures the renormalizability of the theory and makes the theory realistic by giving masses to particles, such as gauge and matter fields, through the vacuum expectation value of the Higgs boson field. The standard model in particle physics also involves the Higgs mechanism and shows remarkable agreement with existing data. After the discovery of the top quark in 1994, the only undetected particle in the standard model is the Higgs boson. Now, studies concerning the Higgs boson search are being conducted in both theoretical and experimental settings and it is expected that the Higgs boson will be discovered within a decade in future experiments at Fermi-Lab and CERN.

Many models (most notably, the technicolor model, the Kaluza-Klein model and recently the approach based on noncommutative differential geometry (NCG) on the discrete) have been constructed for the purpose of understanding the Higgs mechanism. Among these, the NCG approach, originally proposed by Connes [1], provides a unified picture of gauge and Higgs fields as a generalized connection on the principal bundle with the base space $M_{4} \times Z_{N}$. It should be noted that the NCG approach does not demand any physical modes other than the usual one.

Many versions of the NCG approach have appeared since Connes's original presentation, and the standard model has been successfully reconstructed using these approaches. The characteristic feature of the reconstruction of the gauge theory in the NCG approach is to impose restrictions on the gauge and the Higgs quartic coupling constants. This is because the gauge and Higgs fields are represented together as a generalized gauge field. These restrictions yield numerical estimates of the Weinberg angle and mass relations involving the gauge boson and other particles, such as the Higgs boson and top quark in tree level. Several works have appeared [6, 6, 8] estimating the quantum effects of these relations by assuming them to hold at some renormalization point.

The present author has also proposed an unique formulation based on a NCG [3, 5]. Our formulation using a NCG employs a generalization of the usual differential geometry on an ordinary manifold to the discrete manifold $M_{4} \times Z_{N}$. The reconstruction of $\mathrm{SO}(10)$ GUT and the left-right symmetric gauge model

*e-mail address: okum@isc.chubu.ac.jp 
[4] had already been performed using our NCG scheme. In a NCG on $M_{4} \times Z_{2}$, the extra differential oneform $\chi$ in $Z_{2}$ is introduced in addition to the usual one-form $d x^{\mu}$ in $M_{4}$, and therefore our formulation is very similar to ordinary differential geometry, contrastingly, in Connes' original scheme the Dirac matrices $\gamma_{\mu}$ and $\gamma_{5}$ are used to describe the generalized gauge field. In a NCG, the gauge field and the Higgs boson field are given as coefficients of $d x^{\mu}$ and $\chi$, respectively, in the generalized connection on $M_{4} \times Z_{2}$. However, there is no symmetry to mix $d x^{\mu}$ and $\chi$, and, therefore, the ordinary gauge field cannot be transformed into the Higgs boson field. In Ref. [5], the reconstruction of the standard model is successfully carried out based on a new scheme of our NCG. Three generations of fermions, including left and right-handed quarks and leptons, are incorporated. In addition, the strong interaction is nicely included in this scheme. The relations $g^{2}=(5 / 3) g^{\prime 2}=4 \lambda$ are introduced in Ref. [5, where $g, g^{\prime}$ and $\lambda$ are $\mathrm{SU}(2)_{\mathrm{L}}$ and $\mathrm{U}(1)_{\mathrm{Y}}$ gauge coupling constants and the Higgs quartic coupling constant, respectively. However, the grand unification for gauge coupling constants is not achieved in this scheme. The former part of this relation leads to $\sin ^{2} \theta_{\mathrm{W}}=3 / 8$, and the latter part leads to the mass relation $m_{\mathrm{H}}=\sqrt{2} m_{\mathrm{W}}$. We assume that the relations $g^{2}=(5 / 3) g^{\prime 2}=4 \lambda$ hold at one renormalization point. With this assumption, we can perform the renormalization group analysis of the running coupling constants $g, g^{\prime}$ and $\lambda$, and the physical Higgs boson mass can thus be estimated. Within the one loop approximation, this analysis was carried out in Ref. 8. In that context, we found $m_{\mathrm{H}}=164.01 \mathrm{GeV}$ for $m_{\mathrm{top}}=175 \mathrm{GeV}$. In this article, we will perform the two loop analysis of the Higgs boson mass using the same method.

\section{The review of our NCG on $M_{4} \times Z_{2}$}

We first briefly review our previous formulation [5], because it is not well-known among particle physicists. For a detailed description, we refer the reader to Ref. [0].

Let us start with the equation of the generalized gauge field $\mathcal{A}(x, y)$ on the principal bundle with the base space $M_{4} \times Z_{2}$,

$$
\mathcal{A}(x, y)=\sum_{i} a_{i}^{\dagger}(x, y) \boldsymbol{d} a_{i}(x, y)+\sum_{j} b_{j}^{\dagger}(x, y) \boldsymbol{d} b_{j}(x, y),
$$

where $a_{i}(x, y)$ and $b_{j}(x, y)$ are square-matrix-valued functions and are taken so as to commute with each other, because $\sum_{i} a_{i}^{\dagger}(x, y) \boldsymbol{d} a_{i}(x, y)$ is the flavor sector, including the flavor gauge and the Higgs fields, while $\sum_{j} b_{j}^{\dagger}(x, y) \boldsymbol{d} b_{j}(x, y)$ corresponds to the color sector. The indices $i$ and $j$ are variables of the extra internal space which we cannot identify. The operator $\boldsymbol{d}$ in Eq. (2.1) is the generalized exterior derivative defined as follows:

$$
\begin{aligned}
& \boldsymbol{d}=d+d_{\chi}, \\
& d a_{i}(x, y)=\partial_{\mu} a_{i}(x, y) d x^{\mu}, \\
& d_{\chi} a_{i}(x, y)=\left[-a_{i}(x, y) M(y)+M(y) a_{i}(x,-y)\right] \chi, \\
& d b_{j}(x, y)=\partial_{\mu} b_{j}(x, y) d x^{\mu}, \\
& d_{\chi} b_{j}(x, y)=0 .
\end{aligned}
$$

Here, $d x^{\mu}$ is the ordinary one-form basis taken to be dimensionless in Minkowski space $M_{4}$, and $\chi$ is the one-form basis also assumed to be dimensionless in the discrete space $Z_{2}$. We have introduced the $x$-independent matrix $M(y)$ whose hermitian conjugation is given by $M(y)^{\dagger}=M(-y)$. The matrix $M(y)$ determines the scale and pattern of the spontaneous breakdown of the gauge symmetry. Thus, Eq.(2.6) implies that the color symmetry of the strong interaction does not break spontaneously.

Using the algebraic rules in Eqs.(2.2)-(2.6) and the shifting rule invoked in Ref. [5], $\mathcal{A}(x, y)$ can be rewritten as

$$
\mathcal{A}(x, y)=A_{\mu}(x, y) d x^{\mu}+\Phi(x, y) \chi+G_{\mu}(x) d x^{\mu},
$$

where

$$
\begin{aligned}
A_{\mu}(x, y) & =\sum_{i} a_{i}^{\dagger}(x, y) \partial_{\mu} a_{i}(x, y) \\
\Phi(x, y) & =\sum_{i} a_{i}^{\dagger}(x, y)\left(-a_{i}(x, y) M(y)+M(y) a_{i}(x,-y)\right) \\
= & a_{i}^{\dagger}(x, y) \partial_{y} a_{i}(x, y)
\end{aligned}
$$




$$
G_{\mu}(x)=\sum_{j} b_{j}^{\dagger}(x) \partial_{\mu} b_{j}(x)
$$

The functions $A_{\mu}(x, y), \Phi(x, y)$ and $G_{\mu}(x)$ here are identified with the gauge field in the flavor symmetry, Higgs fields, and the color gauge field responsible for the strong interaction, respectively. The gauge transformations of these fields are well defined in the usual manner and it follows that

$$
H(x, y)=\Phi(x, y)+M(y)
$$

is an un-shifted Higgs field whereas $\Phi(x, y)$ denotes a shifted Higgs field with vanishing vacuum expectation value. The nilpotency of $\boldsymbol{d}$ is proved using Eqs.(2.2)-(2.6) along with another algebraic rule in Ref. [5].

With these considerations, we can construct the gauge covariant field strength,

$$
\mathcal{F}(x, y)=F(x, y)+\mathcal{G}(x)
$$

where $F(x, y)$ and $\mathcal{G}(x)$ are the field strengths of flavor and color gauge fields, respectively, and given as

$$
\begin{aligned}
& F(x, y)=\boldsymbol{d} A(x, y)+A(x, y) \wedge A(x, y), \\
& \mathcal{G}(x)=d G(x)+g_{s} G(x) \wedge G(x) .
\end{aligned}
$$

Using the algebraic rules defined in Eqs.(2.2)-(2.6), we have

$$
\begin{aligned}
F(x, y)= & \frac{1}{2} F_{\mu \nu}(x, y) d x^{\mu} \wedge d x^{\nu} \\
& +D_{\mu} \Phi(x, y) d x^{\mu} \wedge \chi+V(x, y) \chi \wedge \chi
\end{aligned}
$$

where

$$
\begin{gathered}
F_{\mu \nu}(x, y)=\partial_{\mu} A_{\nu}(x, y)-\partial_{\nu} A_{\mu}(x, y) \\
+\left[A_{\mu}(x, y), A_{\mu}(x, y)\right], \\
D_{\mu} \Phi(x, y)=\partial_{\mu} \Phi(x, y)+A_{\mu}(x, y)(M(y)+\Phi(x, y)) \\
-(\Phi(x, y)+M(y)) A_{\mu}(x,-y), \\
V(x, y)=(\Phi(x, y)+M(y))(\Phi(x,-y) \\
+M(-y))-Y(x, y) .
\end{gathered}
$$

The quantity $Y(x, y)$ in Eq. 2.17) is auxiliary field and expressed as

$$
Y(x, y)=\sum_{i} a_{i}^{\dagger}(x, y) M(y) M(-y) a_{i}(x, y) .
$$

This function may become a constant field in the present construction. In contrast to $F(x, y), \mathcal{G}(x)$ is simply denoted as

$$
\begin{aligned}
\mathcal{G}(x)= & \frac{1}{2} G_{\mu \nu}(x) d x^{\mu} \wedge d x^{\nu} \\
= & \frac{1}{2}\left\{\partial_{\mu} G_{\nu}(x)-\partial_{\nu} G_{\mu}(x)\right. \\
& \left.\quad+g_{s}\left[G_{\mu}(x), G_{\mu}(x)\right]\right\} d x^{\mu} \wedge d x^{\nu} .
\end{aligned}
$$

With the same metric structure as in Ref. [5] we can obtain the gauge invariant Yang-Mills-Higgs Lagrangian (YMH)

$$
\begin{aligned}
\mathcal{L}_{\mathrm{YMH}}(x)= & -\operatorname{Tr} \sum_{y= \pm} \frac{1}{\tilde{g}^{2}}<\mathcal{F}(x, y), \mathcal{F}(x, y)> \\
= & -\operatorname{Tr} \sum_{y= \pm} \frac{1}{2 \tilde{g}^{2}} F_{\mu \nu}^{\dagger}(x, y) F^{\mu \nu}(x, y) \\
& +\operatorname{Tr} \sum_{y= \pm} \frac{1}{\tilde{g}^{2}}\left(D_{\mu} \Phi(x, y)\right)^{\dagger} D^{\mu} \Phi(x, y)
\end{aligned}
$$




$$
\begin{aligned}
& -\operatorname{Tr} \sum_{y= \pm} \frac{1}{\tilde{g}^{2}} V^{\dagger}(x, y) V(x, y) \\
& -\operatorname{Tr} \sum_{y= \pm} \frac{1}{2 \tilde{g}^{2}} G_{\mu \nu}^{\dagger}(x) G^{\mu \nu}(x) .
\end{aligned}
$$

Here, Tr denotes the trace over internal symmetry matrices including the color, flavor symmetries and generation space. The third term on the right-hand side is the potential term of the Higgs particle.

\section{An numerical estimation of the Higgs boson mass}

In reconstructing the standard model in the present scheme, three generations of left and right-handed quarks and leptons together with the strong interaction must be taken into account. A characteristic point of this formulation is to take the left and right-handed fermions $\psi(x, y)$ with arguments $x$ and $y(= \pm)$ in $M_{4}$ and $Z_{2}$, respectively, as

$$
\psi(x,+)=\left(\begin{array}{c}
u_{\mathrm{L}}^{r} \\
u_{\mathrm{L}}^{g} \\
u_{\mathrm{L}}^{b} \\
\nu_{\mathrm{L}} \\
d_{\mathrm{L}}^{r} \\
d_{\mathrm{L}}^{g} \\
d_{\mathrm{L}}^{b} \\
e_{\mathrm{L}}
\end{array}\right), \quad \psi(x,-)=\left(\begin{array}{c}
u_{\mathrm{R}}^{r} \\
u_{\mathrm{R}}^{g} \\
u_{\mathrm{R}}^{b} \\
0 \\
d_{\mathrm{R}}^{r} \\
d_{\mathrm{R}}^{g} \\
d_{\mathrm{R}}^{b} \\
e_{\mathrm{R}}
\end{array}\right),
$$

where the subscripts $\mathrm{L}$ and $\mathrm{R}$ denote the left-handed and right-handed fermions and the superscripts $r, g$ and $b$ represent the color indices. It should be noted that $\psi(x, y)$ has an index for the three generations, as do the explicit expressions for fermions on the right-hand sides of Eq. (3.1). Thus, $\psi(x, \pm)$ is a vector in the 24-dimensional space. In order to construct the Dirac Lagrangian of the standard model corresponding to $\psi(x, \pm)$ in Eqs.(3.1), we need a 24-dimensional generalized covariant derivative composed of gauge and Higgs fields on $M_{4} \times Z_{2}$. The gauge fields $A_{\mu}(x, y)$ and $G_{\mu}(x)$ in this covariant derivative must constitute the differential representation of the fermion fields in Eqs.(3.1), and therefore they are expressed in $24 \times 24$ matrix forms. The Higgs field $\Phi(x, y)$ is also taken to give the correct Yukawa interaction in the Dirac Lagrangian and is expressed as a $24 \times 24$ matrix ( see Ref. [5] for details). We find Yang-Mills-Higgs Lagrangian for the standard model as follows:

$$
\begin{aligned}
\mathcal{L}_{\mathrm{YMH}}= & -\frac{1}{4} \sum_{k=1}^{3}\left(F_{\mu \nu}^{k}\right)^{2}-\frac{1}{4} B_{\mu \nu}^{2} \\
& +\left|D_{\mu} h\right|^{2}-\lambda\left(h^{\dagger} h-\mu^{2}\right)^{2} \\
& -\frac{1}{4} \sum_{a=1}^{8} G_{\mu \nu}^{a}{ }^{\dagger} G^{a \mu \nu},
\end{aligned}
$$

where

$$
\begin{aligned}
& F_{\mu \nu}^{k}=\partial_{\mu} A_{\nu}^{k}-\partial_{\nu} A_{\mu}^{k}+g \epsilon^{k l m} A_{\mu}^{l} A_{\nu}^{m}, \\
& B_{\mu \nu}=\partial_{\mu} B_{\nu}-\partial_{\nu} B_{\mu}, \\
& D^{\mu} h=\left[\partial_{\mu}-\frac{i}{2}\left(\sum_{k} \tau^{k} g A_{L \mu}^{k}+\tau^{0} g^{\prime} B_{\mu}\right)\right] h, \\
& h=\left(\begin{array}{c}
\phi^{+} \\
\phi_{0}+\mu
\end{array}\right) \\
& G_{\mu \nu}^{a}=\partial_{\mu} G_{\nu}^{a}-\partial_{\nu} G_{\mu}^{a}+g_{c} f^{a b c} G_{\mu}^{b} G_{\nu}^{c},
\end{aligned}
$$

with the following restrictions for coupling constants:

$$
g^{2}=\frac{\tilde{g}^{2}}{12}, \quad g^{\prime 2}=\frac{\tilde{g}^{2}}{20}, \quad \lambda=\frac{\tilde{g}^{2}}{48}, \quad g_{c}^{2}=\frac{g_{s}^{2} \tilde{g}^{2}}{12} .
$$


Equation (3.7) leads to the relation between coupling constants:

$$
g^{2}=\frac{5}{3} g^{\prime 2}=4 \lambda \neq g_{c}^{2}
$$

which implies that the weak, electromagnetic and Higgs quartic coupling constants become equal and also yields $\sin ^{2} \theta_{\mathrm{w}}=3 / 8$ for the Weinberg angle as well as the mass relations

$$
m_{\mathrm{w}}^{2}=\frac{1}{4} g^{2} v^{2}, \quad m_{\mathrm{z}}^{2}=\frac{2}{5} g^{2} v^{2}, \quad m_{\mathrm{H}}^{2}=\frac{1}{2} g^{2} v^{2} .
$$

with the vacuum expectation value of the Higgs boson $v$. These relations hold only at tree level. Here, we assume that these relations hold at a renormalization point and consider their quantum effects by use of the renormalization group (RG) equations.

With the notation

$$
\alpha_{3}=\frac{g_{c}^{2}}{4 \pi}, \quad \alpha_{2}=\frac{g^{2}}{4 \pi}, \quad \alpha_{1}=\frac{5}{3} \frac{g^{\prime 2}}{4 \pi}, \quad \alpha_{\mathrm{H}}=\frac{\lambda}{4 \pi}
$$

for $\mathrm{SU}(3)_{c}, \mathrm{SU}(2)_{\mathrm{L}}$ and $\mathrm{U}(1)_{\mathrm{Y}}$ gauges and the Higgs quartic coupling constants, respectively, the RG equations for these coupling constants are expressed as

$$
\mu \frac{\partial \alpha_{i}}{\partial \mu}=\beta_{i},(i=1,2,3), \quad \mu \frac{\partial \alpha_{\mathrm{H}}}{\partial \mu}=\beta_{\mathrm{H}},
$$

where the $\beta$-functions in the right hand sides are given in Ref. 9 in the two loop approximation. The Yukawa coupling constants of quarks written in $3 \times 3$ matrix form in three generations are included in these $\beta$-functions. We now assume the top quark mass is dominant in the evaluation of the RG equations. Masses of all particles in the standard model are introduced through the vacuum expectation value $v$ of the Higgs field. In this context, the running masses of gauge and Higgs bosons are defined as

$$
m_{\mathrm{W}}=\sqrt{\pi \alpha_{2}} v, \quad m_{\mathrm{H}}=\sqrt{8 \pi \alpha_{\mathrm{H}}} v .
$$

The top quark mass is also expressed as

$$
m_{\mathrm{top}}=\sqrt{2 \pi \alpha_{\mathrm{Y}}} v,
$$

with the Yukawa coupling constant $\alpha_{\mathrm{Y}}$ whose RG equation is also given in Ref. [9]. The RG equation for $v$ is also given in Ref. [9] in the two loop approximation.

The RG equations for $\alpha_{i}(i=1,2,3), \alpha_{\mathrm{H}}, \alpha_{\mathrm{Y}}$ and $v$ are highly non-linear equations with complicated coupling. In order to solve these equations, we need six conditions. Four of these conditions, those for $\alpha_{i}(i=1,2,3)$ and $v$, are given experimentally as [9]

$$
\begin{aligned}
& \alpha_{1}(0)=0.017, \quad \alpha_{2}(0)=0.034, \quad \alpha_{3}(0)=0.12, \\
& v(0)=246 \mathrm{GeV},
\end{aligned}
$$

at $\mu=m_{\mathrm{z}}$ with the variable $t=\log \left(\mu / m_{\mathrm{z}}\right)$. That is, these conditions are given at the neutral gauge boson mass $m_{\mathrm{z}}=91.187 \mathrm{GeV}$. According to Eq.(3.13), the physical top quark mass $m_{\text {top }}$ satisfies the equation

$$
m_{\mathrm{top}}=\sqrt{2 \pi \alpha_{\mathrm{Y}}\left(t_{\mathrm{top}}\right)} v\left(t_{\mathrm{top}}\right),
$$

where $t_{\mathrm{top}}=\log \left(m_{\mathrm{top}} / m_{\mathrm{z}}\right)$. This equation constitutes one condition to solve the RG equations. The remaining condition is

$$
\alpha_{2}\left(t_{0}\right)=\alpha_{1}\left(t_{0}\right)=4 \alpha_{\mathrm{H}}\left(t_{0}\right),
$$

where $t_{0}$ is a constant, fixed in the numerical calculations. The value of $t_{0}$ determines the energy at which the weak, electromagnetic and Higgs quartic interactions are unified. With these considerations, we can find the running Higgs boson mass from Eq.(3.12) as

$$
m_{\mathrm{H}}(t)=\sqrt{8 \pi \alpha_{\mathrm{H}}(t)} v(t) .
$$

The physical Higgs boson mass $m_{\mathrm{H}}$ is determined by imposing the condition that

$$
m_{\mathrm{H}}=\sqrt{8 \pi \alpha_{\mathrm{H}}\left(t_{\mathrm{H}}\right)} v\left(t_{\mathrm{H}}\right)
$$


with $t_{\mathrm{H}}=\log \left(m_{\mathrm{H}} / m_{\mathrm{Z}}\right)$.

The top quark mass has a considerable effect on the Higgs boson mass through Eq.(3.15). The top quark mass $m_{\text {top }}$ reported in a review regarding work on the top quark [10] is given as

$$
m_{\text {top }}=175 \pm 6 \mathrm{GeV} .
$$

We investigated the Higgs boson mass by varying the top quark mass in the range of Eq.(3.19). Table 1. shows the physical Higgs boson mass versus the top quark mass. Compared with the one loop analysis of the Higgs boson mass in Ref. [8], two loop effects lower the numerical values by around $6 \mathrm{GeV}$ because, for example, $m_{\mathrm{H}}=164.01 \mathrm{GeV}$ for $m_{\mathrm{top}}=175 \mathrm{GeV}$ in the one loop analysis. This difference seems crucial since a Higgs boson with mass below $160 \mathrm{GeV}$ could not decay into $\mathrm{W}^{+} \mathrm{W}^{-}$and also experiments designed to search for the Higgs boson depend greatly upon the present analysis. It should be noted that owing to the unitarity requirement, $m_{\text {top }}$ cannot exceed $190 \mathrm{GeV}$ because $\lambda$ would become minus at a much higher value of $t$ in such a case.

It is interesting to investigate the running of the gauge and Higgs quartic coupling constants, because these coupling constants are unified at a point $t_{0}$ as shown in Eq.(3.16). FIG. 1 displays the running of the three coupling constants.

Table 1. Higgs boson mass versus top quark mass.

\begin{tabular}{|c|c|}
\hline top quark $(\mathrm{GeV})$ & Higgs boson $(\mathrm{GeV})$ \\
\hline 169.34 & 150.25 \\
\hline 170.39 & 151.50 \\
\hline 171.74 & 153.40 \\
\hline 172.68 & 154.76 \\
\hline 173.63 & 156.12 \\
\hline 174.34 & 157.14 \\
\hline 175.04 & 158.18 \\
\hline 176.44 & 160.24 \\
\hline 177.37 & 161.61 \\
\hline 178.29 & 163.00 \\
\hline 179.65 & 165.07 \\
\hline 180.34 & 166.12 \\
\hline 181.02 & 167.15 \\
\hline
\end{tabular}

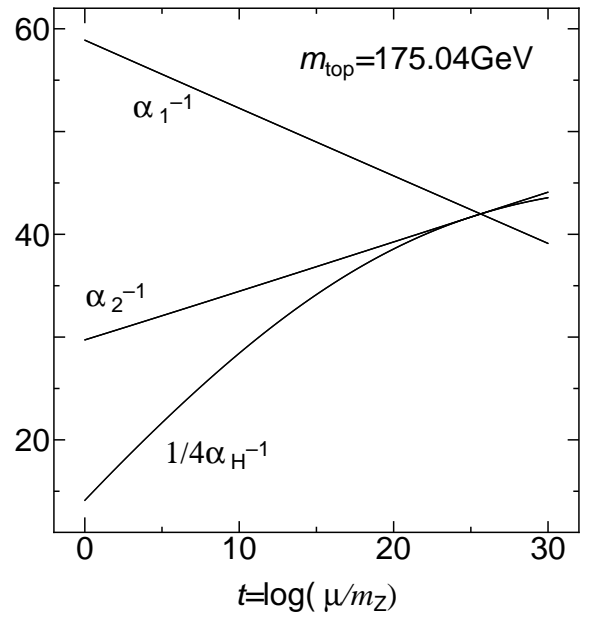

Fig. 1: The running of the three coupling constants.

\section{Conclusion}

In this paper, we have determined the Higgs boson mass by numerically solving the renomalization group equations with the relation between coupling constants Eq.(3.8). It is introduced in the reconstruction of the standard model based on our new scheme of NCG [5]. We assumed that Eq. (3.8) holds at a renormalization point $t_{0}$. However, this leads to an interesting result that the weak, electromagnetic and Higgs quartic coupling constants become equal at $t_{0}$ as shown Fig.1. In the case of $t_{\text {top }}=175.04$, $t_{0}=26.635$ which amounts to $\mu=3.37 \times 10^{13} \mathrm{GeV}$.

We obtain $150.25 \leq m_{\mathrm{H}} \leq 167.15$ in the range of top quark mass $169.34 \leq m_{\mathrm{top}} \leq 181.02$. We hope that this result will be useful for experiments searching for the Higgs boson.

\section{References}

[1] A. Connes, p.9 in The Interface of Mathematics and Particle Physics, ed. D. G. Quillen, G. B. Segal, and Tsou. S. T., Clarendon Press, Oxford, 1990. See also, Alain Connes and J. Lott, Nucl. Phys. B (Proc. Suppl.) 18B, 57 (1990).

[2] References in J. Madore and J. Mourad, hep-th/9601169. 
[3] Y. Okumura, Prog. Theor. Phys., 96, 1021 (1996).

[4] Y. Okumura, Phys. Rev. D, 50 (1994), 1026, Prog. Theor. Phys.,94 (1995), 589, hep-th/9703213 to appear in Nuovo Cimento A.

[5] Y. Okumura, Prog. Theor. Phys. 95, 969 (1996).

[6] E. Alvarez, J.M. Gracia-Bondía and C.P. Martín, Phys. Lett., B306, 55 (1993); ibit. B329, 259 (1994).

[7] T. Shinohara, K. Nishida, H. Tanaka and I.S. Sogami, Prog. Theor. Phys. 96 , 1179 (1996).

[8] Y. Okumura, hep-ph/9608208

[9] H. Arason, D.J. Castaño, B. Kesthelyi, S. Mikaelian, E.J. Piard, P. Ramond, Phys. Rev. D 46, 3945 (1992); M.E. Machacek and M.T. Vaughn, Phys. Lett. 103B, 427 (1981); Nucl. Phys. B222, 83 (1983); ibit. B136, 221 (1984); ibit. B249, 70 (1985).

[10] S. Willenbrock, hep-ph/9611240. 